\title{
Modeling and Simulation on
}

\section{Desynchronization of Neuronal Networks under Electrostatic Field}

\author{
WANG Rui-Quan \\ Qingdao No. 2 Middle School of Shangdong \\ Province, \\ Qingdao, 266100, China \\ black-rasion-detre@chu2byo.com
}

\author{
WANG Huai-Yang \\ Information science and engineering school, \\ Ocean University of China , \\ Qingdao,266100, China \\ wanghuaiyang99@yahoo.com
}

\begin{abstract}
To explore therapeutic mechanism for Parkinson's disease and other shivering senile diseases, the effect of external electrostatic field on the spiking of single Izhikevich neuron model and synchronized firing of large scale Izhikevich neuronal network were studied with computer simulation. The simulation results suggest that external electrostatic field can decrease the firing rate of single neuron and restrain the firing synchronism of the large scale neuronal networks, and real-time simulation for firing of large scale neuronal networks was realized with proposed method in the study. The conclusion may explain therapeutic effect of external electric fields on Parkinson's disease and other shivering diseases.
\end{abstract}

Keywords-simulation; neuron; neuronal network; synchronization; desynchronization

\section{INTRODUCTION}

At present, the electromagnetic field is used for the treatment of neurological diseases in invasive and noninvasive ways. Transcranial magnetic stimulation (TMS) is a noninvasive method of treatment, while a weak, slowly changing magnetic field is applied to the brain, weak electric field will be induced at the specific part of brain, and then treatment effect will be produced. Deep brain stimulation (DMS) is a kind of invasive treatment method, as leads are implanted at a specific part in the brain, electric field is formed in the brain to change the abnormal electrical activity. This method has remarkable effect for the treatment of Parkinson's disease, tremor, and other neuronal discharge diseases caused by excessive synchronization. In order to investigate the mechanism of electric field treatment, it is critical to study the influence of electric field on the synchronous firing of neurons and firing synchronism in neuronal network.

Lu Qishao team[1-4] used the coupled oscillator theory in theoretical mechanics to study the dynamics of coupled neurons, paying attention to the research on synchronization among neurons, found the dynamics and transformation produced in coupled neuron system change with key parameters of the system. The defect is that most of their results are merely for synchronization between two neurons. Wang Jiang team[5-8] investigated influence of external electric field on neuronal firing, and got the rules of induced electric field in the brain on the firing neuron and neuronal network. The disadvantages are that the simulating models are too complex in computation to realize real-time description of firing activity in large-scale neuronal network, and the effect of external electric field on the synchronous firing of large-scale neural network had not be revealed. To investigate the influence of external electric field on the firing synchronism of neurons and realize the real time characterizing of firing in large scale neuronal networks, Izhikevich neuron model[9], which is simple in computation, is used in the study to consider the effect of external electrostatic field on the firing of single neuron, and then simulate the changing of firing synchronism of the neuronal 
network, which consists of 1000 neurons that are connected with random connection strength and in all-all connection pattern. The results show that proper electrostatic field can reduce the firing synchronism in the large scale neuronal networks.

\section{MODIFIED IZHIKEVICH NEURAL MODEL}

According to the literature[6], a depolarization induced voltage $\triangle v$ will be emerge across the neuronal membrane while the brain is under external electrostatic field. When the induced field is static DC field whose amplitude is E, the depolarization induced voltage $\triangle v$ across the cell membrane is also DC, i.e., $\Delta v=\lambda E$. The polarization length is taken as $\lambda=1 \mathrm{~mm}$ to study conveniently, that is the value of DC electrostatic field equals that of depolarization induced voltage.

Then, total neuronal membrane potential becomes $v_{m}=v+\Delta v$. The influence of external electrostatic field just induces a DC transmembrane voltage, and does not change the basic structure of the computational model. Consequently, the modified Izhikevich model describing the single neuron under external electrostatic field is as follows:

$$
\begin{aligned}
v^{\prime} & =0.04(v+\Delta v)^{2}+5(v+\Delta v)+140-u+I \\
u^{\prime} & =a(b v-u)
\end{aligned}
$$

The resetting after firing is as in its original form:

$$
\begin{aligned}
& \text { If } \mathrm{v} \geq 30 \text {, then } \\
& v=c \\
& u=u+d
\end{aligned}
$$

The large scale neuronal network model is modified as:

$$
\begin{aligned}
& v_{i}^{\prime}=0.04\left(v_{i}+\Delta v\right)^{2}+5\left(v_{i}+\Delta v\right)+140 \\
&-u_{i}+I_{i}+\sum_{j} s_{i j} \delta_{j}\left(t-t^{\prime}\right) \\
& u_{i}^{\prime}=a_{i}\left(b_{i} v_{i}-u_{i}\right)
\end{aligned}
$$

If $v_{i} \geq 30$, then

$$
\begin{gathered}
v_{i}=c_{i} ; \\
u_{i}=u_{i}+d_{i}
\end{gathered}
$$

\section{EXPERIMENT RESULTS}

\section{A. A. Effect of external electrostatic field on firing of single} neurons

To investigate the influence of external electrostatic field on the firing of single neurons, modified Izhikevich neural model is solved with Euler method in computational mathematics, time step is selected as $1 \mathrm{~ms}$, and computing process is realized with Matlab program. The membrane potentials under external electrostatic fields with different intensity are computed, and then firing rates are determined. The firing rate curve changing with electric field intensity is shown in Fig. 1.

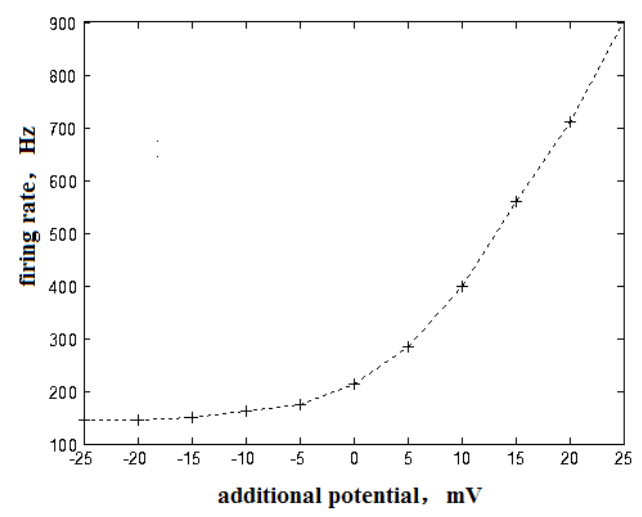

Fig.1 Effect of external electrostatic field on the firing rate of a single neuron

Changing trend of neuron firing rate under the electric field can be seen from the figure. Comparing with the condition without external field, firing rate decreases with the amplitude of negative attached transmembrane potential, and increases with that of positive attached transmembrane potential. That means firing rate of single neurons can be controlled with proper external electrostatic field. 


\section{B. Effect of external electrostatic field on synchronism of neuronal networks}

To investigate the influence of external electrostatic field on the firing in the large scale neuronal network, modified Izhikevich neuronal network model is solved with Euler method. Firing of neurons during $0 \sim 1000 \mathrm{~ms}$ is simulated with Matlab, if the neuron i fires at time $t$, a black spot is marked at $(t, i)$ in the figure, otherwise, a blank is left at $(t, \mathrm{i})$.
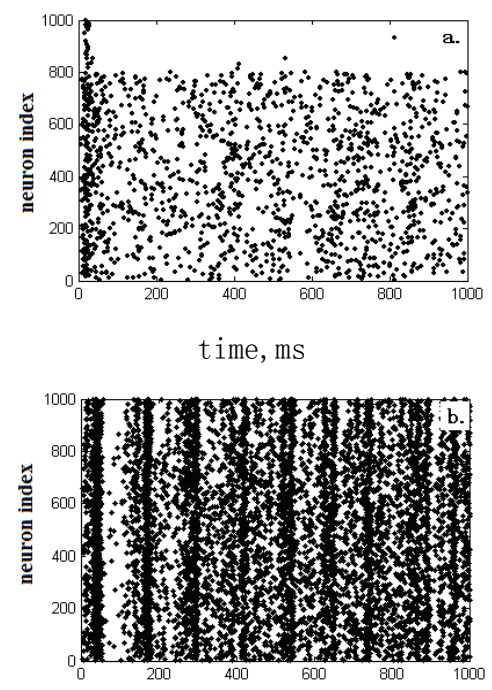

time, ms

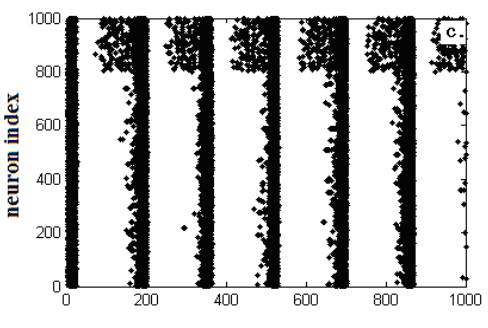

time, ms

Fig.2 Comparison of firing pattern in neuronal network under different electrostatic fields

a. Attached voltage formed by external eletrostatic field is $-10 \mathrm{mV}$;

b. Attached voltage formed by external eletrostatic field is $0 \mathrm{mV}$;

Attached voltage formed by external eletrostatic field is $10 \mathrm{mV}$

While an external electric field is not applied, $10 \mathrm{~Hz}$ alpha synchronous oscillation in the network forms in the first 250 milliseconds, then $40 \mathrm{~Hz}$ gama synchronous oscillation appears, as shown in Fig 2 (b); At the same time the number of synchronously firing neurons at less than 100 in the first 250 milliseconds, and then the number is no more than 40 , as shown in Fig. 3 (b). The applied electrostatic field formed $10 \mathrm{mV}$ additional potential in the neurons of the network, synchronous oscillation does not appear, such as shown in Fig. 2 (a); comparing to the condition no external field, the number of synchronous firing neurons in the range of $0 \sim 1000 \mathrm{~ms}$ greatly decreases, the maximum number is not more than 15 , as shown in Fig. 3 (a).
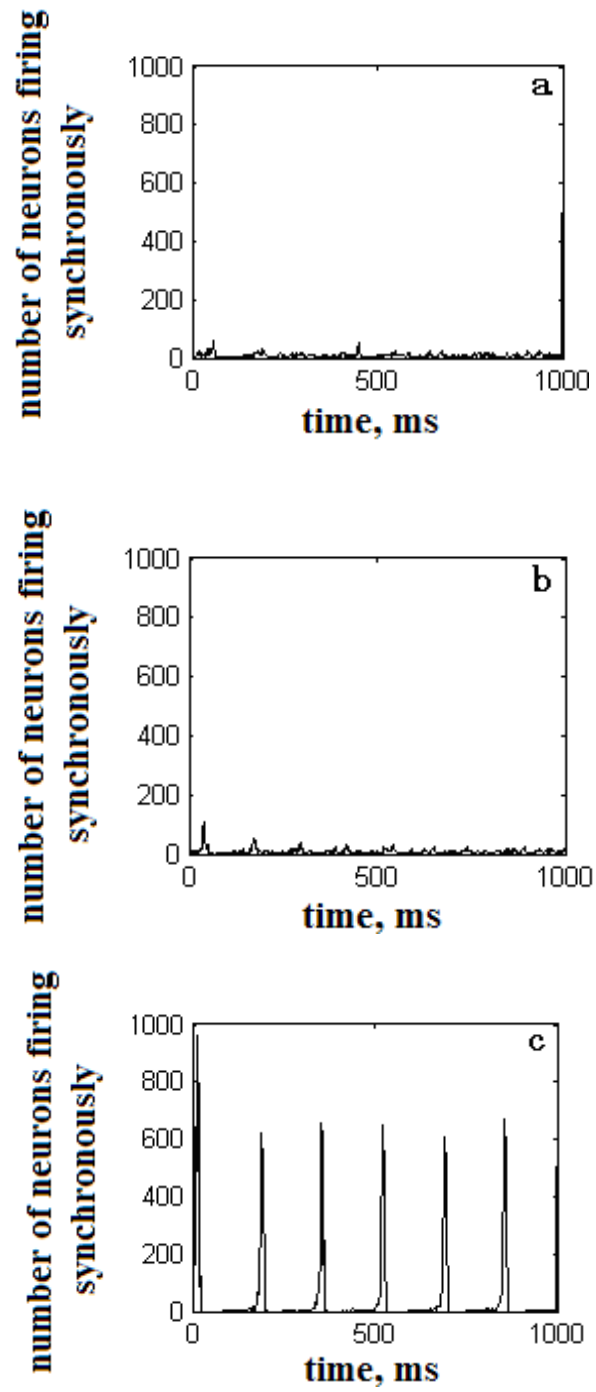

Fig.3 Number of simultaneously firing neurons in neuronal network under different electrostatic fields

a. Attached voltage formed by external eletrostatic field is $-10 \mathrm{mV}$;

b. Attached voltage formed by external eletrostatic field is $0 \mathrm{mV}$;

c. Attached voltage formed by external eletrostatic field is $10 \mathrm{mV}$ 
While the applied electrostatic field formed $+10 \mathrm{mV}$ additional potential in the neurons of the network, there appears extremely obvious synchronizing oscillation in about 6 $\mathrm{Hz}$, as shown in Fig. 2 (c) ; The number of synchronous firing neurons during $0 \sim 1000 \mathrm{~ms}$ is greatly increased compared with no external field, the largest number more than 600, as shown in Fig. 3 (C).

The computer we use is produced by Lenovo Group, its CPU is INTEL(R) Celeron ( $\mathrm{R})$, speed is $2.66 \mathrm{GHz}$, and memory capacity is $504 \mathrm{mb}$. Simulation program is operated with the computer, simulating result will be displayed in less than 1 second. That means the real-time simulation of large scale neuronal networks be realized, so the simulating program may be operated repeatedly. It is helpful for getting large amounts of simulation results.

\section{DISCUSSION}

In this study, through the simulation of a single neuron' firing under static electric field, we can draw the following conclusions: The firing frequency of a single neuron deceases while a negative potential in the neuron is formed with external field and increases while a positive potential formed. For the large scale neural network, as it is not affected by external static electric field, firing of neurons in the network show a certain synchronism, alpha and gamma rhythms emerge, and the number of synchronously firing neurons does not exceed 15; as positive potentials are formed across the neuronal membranes, rhythmicity of firing in the networks obviously enhances, the number of synchronously firing neurons increases sharply; as negative potentials formed, rhythmicity of firing obviously declines. That means a proper external electrostatic field can desynchronize the firing in large scale neuronal networks and the therapeutic mechanism of TBS and DBS on Parkinson disease and shivering senile diseases lie on eliminating morbid synchronism in intracerebral motor neurons that causes the patients trembling pathologically.

\section{V.CONCLUSION}

Modified Izhikevich neuron model is used to simulate the synchronism in the large-scale neuronal networks in the study, the results indicate that the firing rate of single neuron decreases and firing synchronism in the large-scale neuronal networks declines under the proper external electrostatic field, and firing activities in large-scale neuronal networks can be characterized in real-time form.

We verified that external electrostatic field can be used in the field of neural system disease treatment to change the abnormal firing rhythmicity of neurons, which will provide some theoretical basis for the treatment of epilepsy and other shivering diseases.

Next step, we will probe into the effect of chronic alternating electrostatic field on firing of Izhikevich neuron and neuronal network to discuss clinical treatment mechanism of TBS and DBS more detailedly.

[1] Qingyun Wang, Qishao Lu, Guanrong Chen. Bifurcation and synchronization of synaptically coupled FHN models with time delay. Chaos, Solitons \& Fractals, 2009; 39(2):918-925

[2] Xia Shi, Qishao Lu. Burst synchronization of electrically and chemically coupled map-based neurons. Physica A: Statistical Mechanics and its Applications,2009; 388(12): 2410-2419

[3] Fang Han, Qishao Lu. Chaotic burst synchronization in heterogeneous small-world neuronal network with noise. International Journal of Non-Linear Mechanics,2009; 44(3):298-303

[4] Qishao Lu, Zhuoqin Yang. Dynamics and transitions of firing patterns in deterministic and stochastic neuronal systems. Chaos, Solitons \& Fractals, 2009; 40( 2): 577-597

[5] Yi Guo-Sheng, Wang Jiang, Wei Xi-Le et. Dynamical Analysis of Firing Patterns Exhibited by Neuron Exposed to Static Direct Current Electric Field. Journal of Tianjin University, 2012; 45(12):1096-1104

[6] Jin Qi-Tao, Wang Jiang, Yi Guo-Sheng et. Analysis on firing initial dynamic mechanism of maximum neuronal model under TMS external electric field. Acta Phys. Sin., 2012;61(11):517-526

[7] Yu Haitao, Wang Jiang, Deng Bin et. Firing patterns of map-based neuron under extracellular alternating-current field. ACTA BIOPHYSICA SINICA, 2012;26(10):907-918

[8] Yu Kai, Wang Jiang et. Firing patterns of map-based neuron under extracellular alternating-current field. Journal of Tianjin University, 2013;46(8):726-736

[9] Izhikevich, E.M. Simple model of spiking neurons. IEEE Trans Neural Netw, 2003; 14(6):1569-1572. 